\section{Morphea statt Bluterguss}

Was selten ist, wird auch selten erkannt: Im Schnitt dauert es fast ein Jahr, bis Kinder mit einer Morphea die richtige Diagnose erhalten. Gelangen die kleinen Patienten aber erst einmal zum Dermatologen, liegt dieser mit seiner Einschätzung meist richtig. Nach einer aktuellen Studie liegen dann bei der Hälfte der Kinder Symptome wie Fibrose oder Sklerose vor.

Eine ine zirkumskripte Sklerodermie oder Morphea tritt mit einer Inzidenz von nur einer bis drei Erkrankungen pro 100.000 Kinder und Jahr auf. Sie zählt damit zu seltenen dermatologischen Erkrankungen. Kein Wunder also, dass weder Hausärzte noch Pädiater bei Ödemen, Hautrötungen oder Entzündungen an eine Sklerodermie denken. Dermatologen aus Zürich und London haben nun bei 50 Kindern mit Morphea untersucht, was mit ihnen alles geschah, bis sie die richtige Diagnose erhielten. Dazu führte das Team um Dr. Lisa Weibel ausführliche Gespräche mit den Eltern und wertete die Krankenakten der Kinder aus.

Im Schnitt begannen die Symptome im Alter von fünf Jahren, die Eltern vermuteten meist einen Bluterguss, Ekzeme,
Verbrennungen oder eine Hautinfektion. Nach durchschnittlich einem Monat wurden die Kinder zu einem Hausarzt oder Pädiater geschickt, dessen Diagnose in der Regel nicht besser war als die der Eltern. Erst nach siebeneinhalb Monaten sahen die Kinder einen Spezialisten, meist einen Dermatologen, gelegentlich aber auch schon einen Chirurgen. Bei immerhin zwei Drittel der Kinder wurde dabei die richtige Diagnose gestellt. Von den übrigen 18 Kindern bekamen zehn zunächst keine Diagnose, bei zweien wurde eine atopische Dermatitis angenommen, bei einem ein Melanom und bei vier Kindern tippten die Ärzte auf Gefäßveränderungen. 13 dieser Kinder wurden zu einem weiteren Spezialisten geschickt. Nach im Schnitt elf Monaten stand bei allen Kindern die richtige Diagnose fest. Charakteristische Merkmale wie eine Verteilung der Hautveränderungen entlang der BlaschkoLinien sowie eine Haut- und Gewebeatrophie ließen sich bei etwa $80 \%$ der Kinder nachweisen, Fibrose, Sklerose sowie Haarausfall im betroffenen Areal waren bei jeweils 50\% der Patienten zu erkennen.

Die Ärzte um Weibel erinnern daran, dass sich eine Morphea im frühen Stadium mit topischen Arzneien sowie Phototherapie gut behandeln lässt, während in späteren Stadien meist eine systemische Therapie nötig ist und schwere Haut-, Gewebe- und Knochendefekte auftreten können.

Fazit: Mit elf Monaten dauert es noch immer sehr lange, bis eine Morphea erkannt wird. Dies liegt hauptsächlich daran, dass die betroffenen Kinder erst sehr spät vom behandelnden Arzt zu einem Spezialisten überwiesen werden.

Thomas Müller

Weibel $L$ et al. Misdiagnosis and delay in referral of children with localized scleroderma. Br J Dermatol 2011, 165: 1308-13

\title{
Zungenpigment nach Chemotherapie
}

\section{Dass Zytostatika eine Vielzahl von schwerwiegenden Nebenwirkungen aufweisen, ist bekannt. Das große Spektrum der unerwünschten Ereignisse umfasst jedoch auch seltenere Phänomene.}

$\mathrm{N}$ ach brusterhaltender Operation und der Biopsie eines Sentinel-Lymphknotes in der Axilla wegen eines invasiven duktalen Mammakarzinoms unterzog sich eine 42-jährige Frau einer Chemo-

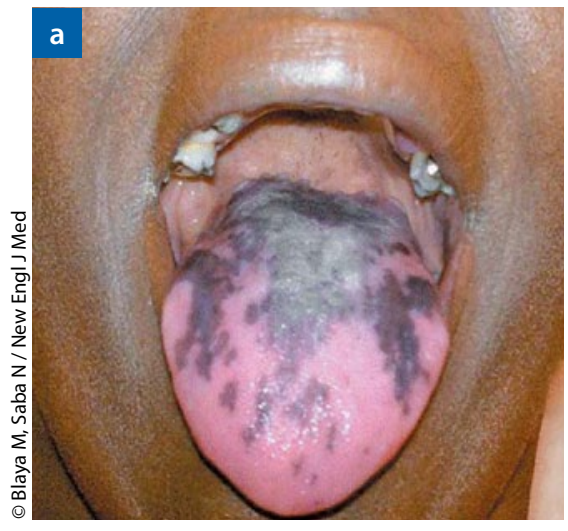

therapie mit Doxorubicin, Cyclophosphamid und Paclitaxel. Nach dem zweiten Zyklus mit Doxorubicin und Cyclophosphamid traten auf der Zunge der Patientin hyperpigmentierte Flecken auf

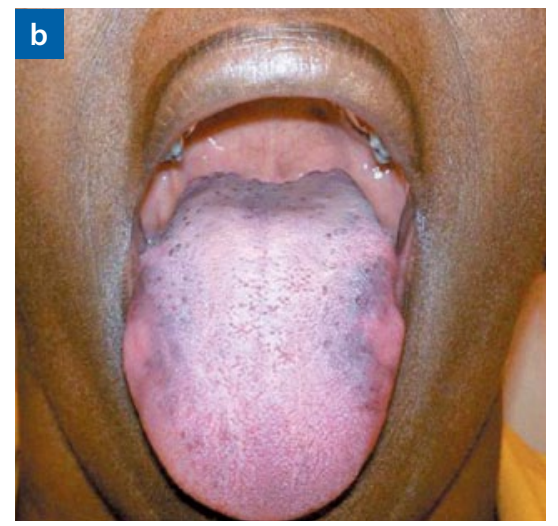

(Abb. a). Handflächen, Fußsohlen, Fingernägel und andere Hautareale waren nicht betroffen.

Fazit: Hyperpigmentierungen der Haut sind eine bekannte Nebenwirkung einer Reihe von Zytostatika und insbesondere bei Anwendung von Doxorubicin und $\mathrm{Cy}$ clophosphamid nicht selten. Die Veränderungen können lokal oder generalisiert sein und Haut, Schleimhäute, Nägel oder Haare betreffen. Die zugrunde liegenden Mechanismen sind unbekannt. Bei dieser Patientin bildeten sich die Veränderungen nach Abschluss der Chemotherapie allmählich zurück und verschwanden nach drei Monaten vollständig (Abb. b).

Prof. Dr. Hermann Füeß।

Blaya M, Saba N. Chemotherapy-indices hyperpigmentation of the tongue. New Engl J Med 2011; 365: 10, e20

Fleckenbildung während der Chemotherapie (a) und Rückbildung nach Therapieende (b). 